\title{
Integrated Transformer-Coupled Balun Bandpass Filters with an Optimal Common-Mode Rejection Ratio
}

\author{
Chien-Hsun Chen, Student Member, IEEE, Chien-Hsiang Huang, Student Member, IEEE, \\ and Tzyy-Sheng Horng, Senior Member, IEEE
}

\begin{abstract}
This paper describes a compact transformercoupled balun bandpass filter design based on integrated circuit technology. Using a planar transformer with high-density fully symmetrical wiring not only greatly reduces the component size, but also provides a superior balance at the differential output ports. In addition, applying the tapped feed-line structure to the balun bandpass filter is accomplished by carefully studying the impedance-matching method and transmission-zero mechanism. Interestingly, the proposed balun bandpass filter can guarantee an optimal common-mode rejection ratio level in a specific operating frequency range by precisely controlling the fractional bandwidth of the filter passband.
\end{abstract}

Index Terms-Balun bandpass filters, common-mode rejection ratio, planar transformer.

\section{INTRODUCTION}

M OST radio frequency (RF) receiver front-ends require a bandpass filter to suppress the input interferences, as well as a balun to convert an input single-ended RF signal into an output-balanced one for subsequent processing with posterior differential active circuits. Fig. 1 schematically depicts a balun bandpass filter with the integrated functions of both a bandpass filter and a balun. A printed circuit board (PCB)-embedded component [1] and a low-temperature cofired ceramic (LTCC) component [2] using a separate design of the bandpass filter and the balun result in a complex architecture and a bulky circuitry space. The interaction between the bandpass filter and the balun degrades the performance in terms of insertion loss and output balance. Several integrated schemes have been developed to achieve a reduced component size and satisfactory performance in recent years. A simple design method in [3] merges the output stage inductor of a filter with a portion of the posterior balun using silicon integrated passive device (IPD) technology. However, the level

Manuscript received April 21, 2011; revised September 27, 2011; accepted October 20, 2011. Date of publication November 24, 2011; date of current version January 5, 2012. This work was supported in part by the National Science Council, Taiwan, under Grant 97-2221-E-110-034-MY3, Grant 972221-E-110-035-MY3, and Grant 100-2622-E-110-001-CC1, and the Department of Industrial Technology, Taiwan, under Grant 99-EC-17-A-01-S1-104. Recommended for publication by Associate Editor M. Cases upon evaluation of reviewers' comments.

The authors are with the Department of Electrical Engineering, National Sun Yat-Sen University, Kaohsiung 804, Taiwan (e-mail: d943010020@student. nsysu.edu.tw; d9131820@student.nsysu.edu.tw; jason@ee.nsysu.edu.tw).

Color versions of one or more of the figures in this paper are available online at http://ieeexplore.ieee.org.

Digital Object Identifier 10.1109/TCPMT.2011.2174440 of size reduction is rather limited because the reduction of component count is not significant in such a lumpedelement structure. In addition to a dual-mode ring resonator [4] requiring an effective length of a transmission line for a balun bandpass filter design, further reducing the size is extremely difficult. Other studies include the three coupled tanks structure [5], the three-pole cross-coupled filter topology [6], the multi-coupled lines configuration with shunt capacitors [7], [8], and the vertical split ring resonator construction [9] that use LTCC technology. In addition, the parallel connected $L C$ resonator architecture is also embedded into an eightlayered PCB [10]. Although the designs [5]-[10] exploit a multilayer structure to reduce the component size with the help of LTCC or PCB build-up technology, the geometry is difficult to maintain symmetry so as to considerably degrade the output balance. Recently, balun bandpass filters using two sets of two symmetric stepped impedance resonators are verified to be well balanced for arbitrary bandwidth [11]. However, the method proposed by [11] is limited to a fourth-order bandpass filter design, which is disadvantageous to the reduction of size and insertion loss.

The authors' previous work [12] presented a transformercoupled bandpass filter design using glass IPD technology. The advantages include very small size and ease of integrating a balun without increasing the component size and loss. In addition, the resultant balun bandpass filter has a significantly higher common-mode rejection ratio (CMRR) level when compared to the available literature. By expanding the results of [12], this paper provides a detailed methodology of the balun bandpass filter designs from the proposed equivalent circuits and a further parametric study of the CMRR. It is interesting to find that by controlling the fractional bandwidth of the passband, we can flexibly adjust the CMRR level to meet diverse applications. Based on this finding, a wideband balun with a demanded high CMRR level is realized in complementary metal-oxide-semiconductor (CMOS) technology by adequately imposing a bandpass filter function with a controlled bandwidth on the balun.

\section{Design OF TRANSFORMER-COUPLED BANDPASS FILTER}

This paper begins with a transformer-coupled bandpass filter design using the coupled resonator synthesis method [13] in frequency band ranging from 2.7 to $2.9 \mathrm{GHz}$ 


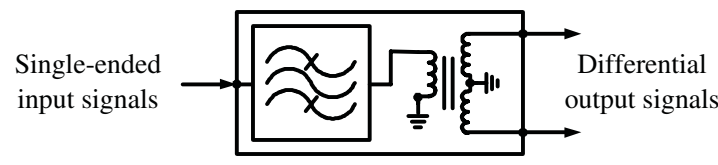

Fig. 1. Block diagram of a balun bandpass filter.

for microwave multipoint distribution system applications. The adopted method can determine the passband bandwidth by accurately estimating the mutual inductance required to couple the primary and secondary coils in the transformer. Fig. 2(a) and (b) illustrates the equivalent circuit of the proposed transformer-coupled bandpass filter with symmetric and antisymmetric feed structure, respectively. The equivalent circuit in Fig. 2(a) or (b) comprises two parallel resonant circuits magnetically coupled with a transformer mutual inductance $M$. Each resonant circuit includes a coil inductance $\left(L_{1}-L_{2}-L_{1}\right)$ resonated with a pair of dual capacitors of capacitance $C$, yielding the following resonant frequency:

$$
\omega_{0}=\sqrt{\frac{2}{\left(2 L_{1}+L_{2}\right) C}} .
$$

In addition, a mutual capacitance $C_{c}$ is used between two resonant circuits, representing the parasitic electrical coupling between the two coil-type resonators.

Two major parameters for applying the coupled resonator synthesis method to the bandpass filter design are the coupling coefficient $k$ between the two resonators and the external quality factor $Q_{e}$ at the input and output. The coupling coefficient depends mainly on the spacing of the interwound turns of the coils in the transformer. For even and odd mode analysis of the equivalent circuits in Fig. 2(a) and (b), the same even and odd resonant frequencies can be found, respectively, as

$$
\begin{aligned}
\omega_{\text {even }} & =\frac{\sqrt{2}}{\sqrt{\left(2 L_{1}+L_{2}-M\right) C}} \\
\omega_{\text {odd }} & =\frac{\sqrt{2}}{\sqrt{\left(2 L_{1}+L_{2}+M\right)\left(C+C_{c}\right)}} .
\end{aligned}
$$

Based on knowledge of the two resonant frequencies, the coupling coefficient can be estimated as

$$
k=\frac{\omega_{\text {even }}^{2}-\omega_{\text {odd }}^{2}}{\omega_{\text {even }}^{2}+\omega_{\text {odd }}^{2}} .
$$

Assuming that $C_{c}$ is small, so that $M C_{c} \ll 2 C\left(2 L_{1}+L_{2}\right)$, the expression (4) can be further approximated as

$$
k \approx \frac{C_{c}}{2 C}+\frac{M}{2 L_{1}+L_{2}}=k_{E}+k_{M} .
$$

The above equation clearly indicates that the mixed coupling between the two resonators arises from the summation of the electrical and magnetic coupling coefficients.

The external quality factor of the input coil-type resonator can be evaluated as a function of the tapped feed position.

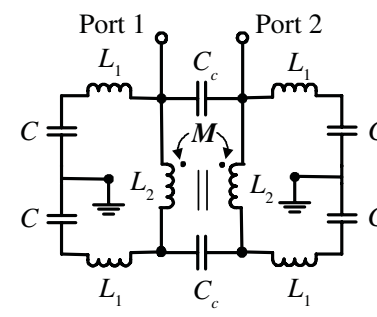

(a)

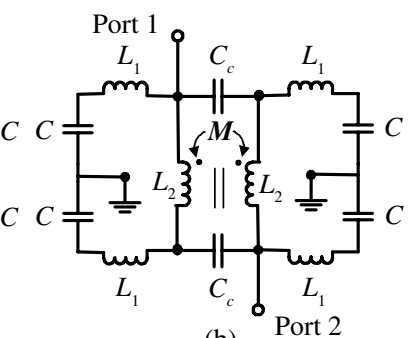

(b)
Fig. 2. Equivalent circuits of the designed transformer-coupled bandpass filters. (a) Symmetric feed structure. (b) Antisymmetric feed structure.

A general formula can be easily derived from the definition given in [14] and is shown as

$$
Q_{\mathrm{e}}=\left.R_{\mathrm{in}} \frac{\omega}{2} \frac{\partial B}{\partial \omega}\right|_{\omega=\omega_{0}}=R_{\mathrm{in}} \frac{\sqrt{8 C\left(2 L_{1}+L_{2}\right)^{3}}}{L_{2}^{2}}
$$

where $R_{\text {in }}$ denotes the reference impedance looking into the source termination at the input coil-type resonator, and $B$ is the total susceptance seen at the feed point looking into the resonator. To match the source impedance, $R_{\text {in }}$ must equal 50 $\Omega$. In this manner, the external quality factor of the output coil-type resonator can be obtained in the same way as in (6).

The coil turn spacing and tapped feed position can be determined by using the relations of $k$ and $Q_{e}$ for the second-order filter prototype elements. These relations can be expressed as [13]

$$
\begin{aligned}
k & =\frac{\Delta}{\sqrt{g_{1} g_{2}}} \\
Q_{\mathrm{e}} & =\frac{g_{0} g_{1}}{\Delta}=\frac{g_{2} g_{3}}{\Delta}
\end{aligned}
$$

where $\Delta$ represents the fractional bandwidth and $g_{i}$ is the $i$ th prototype element value. For a Chebyshev bandpass filter design, the passband insertion loss can be predicted by the formula given in [15] and shown as

$$
I L_{\omega_{0}}(\mathrm{~dB}) \approx \frac{4.343}{\Delta \cdot Q_{\mathrm{u}}}\left(g_{1}+g_{2}\right)
$$

where $Q_{u}$ denotes the unloaded quality factor of a coil-type resonator at the resonant frequency.

In addition to achieving the desired specification in a passband, transmission zeros are extensively adopted to increase either the selectivity or the rejection at stopband for bandpass filter design. A previous work [16] found that a $0^{\circ}$ feed structure has two additional transmission zeros than a non- $0^{\circ}$ feed structure with the same passband response. Based on the concept in [16], this paper demonstrates the effectiveness of two feed structures of symmetrical and antisymmetrical construction, as shown in Fig. 2(a) and (b), respectively. The admittance parameters of $Y_{21}$ for these two feed structures are derived as in (10) and (11), shown at the bottom of the page,

$$
\begin{aligned}
Y_{21, \text { sym }} & =\frac{\left(Z_{m}+Z_{c}\right)\left(2 Z_{1} Z_{m}+Z_{m} Z_{c}-2 Z_{1}^{2}\right)-Z_{2}^{2} Z_{c}-2 Z_{1} Z_{2}\left(Z_{1}+Z_{2}+Z_{c}\right)}{2 Z_{1} Z_{2} Z_{c}\left(Z_{1}+Z_{2}+Z_{c}\right)+Z_{2}^{2} Z_{c}^{2}+Z_{c}\left(Z_{1}-Z_{m}\right)\left(2 Z_{1} Z_{m}+Z_{1} Z_{c}+Z_{m} Z_{c}\right)} \\
Y_{21, \text { anti-sym }}= & \frac{2 Z_{1}\left(Z_{2}+Z_{m}\right)\left(Z_{1}+Z_{c}\right)+Z_{c}\left(2 Z_{1}^{2}+Z_{m} Z_{c}\right)}{Z_{1} Z_{c}\left(Z_{1} Z_{c}+2 Z_{m}^{2}\right)-\left(Z_{1}^{2}+Z_{c}^{2}\right)\left(Z_{2}^{2}-Z_{m}^{2}\right)-2 Z_{1} Z_{c}\left(Z_{1}+Z_{2}\right)\left(Z_{2}+Z_{c}\right)}
\end{aligned}
$$




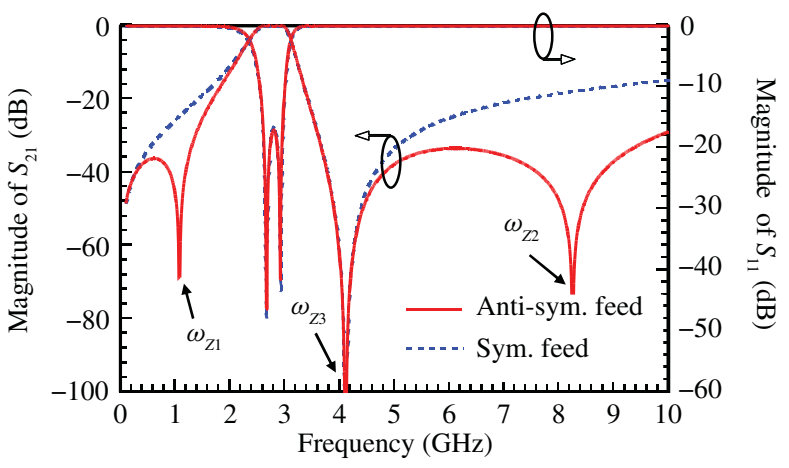

Fig. 3. Comparison of the magnitude of $S_{21}$ and $S_{11}$ of the equivalent circuits with two different feed structures for the transformer-coupled bandpass filter.

where

$$
\begin{aligned}
Z_{1} & =j \omega L_{1}+\frac{1}{j \omega C} \\
Z_{2} & =j \omega L_{2} \\
Z_{m} & =j \omega M \\
Z_{c} & =\frac{1}{j \omega C_{c}} .
\end{aligned}
$$

As is well known, transmission zeros occur at frequencies where the admittance parameter $Y_{21}$ of the two-port bandpass filters equals zero [17]. Fig. 3 compares the magnitudes of $S_{21}$ and $S_{11}$ between the symmetrical and the antisymmetrical feed structures for the equivalent circuits in Fig. 2(a) and (b). The corresponding element values are $L_{1}=1.5 \mathrm{nH}, L_{2}=3.3 \mathrm{nH}$, $M=0.9 \mathrm{nH}, C=1 \mathrm{pF}$, and $C_{c}=0.03 \mathrm{pF}$. Obviously, the antisymmetrical feed structure has two additional transmission zeros of $\omega_{Z 1}$ and $\omega_{Z 2}$ within $10 \mathrm{GHz}$ and can be expressed as

$$
\sqrt{\frac{\omega_{Z 1,2}=}{\frac{\left(A+C M+2 C_{c} L_{1}\right) \pm \sqrt{(A+C M)^{2}-4 C_{c} L_{1}(A-C M)}}{2 A L_{1}\left(C+C_{c}\right)}}}
$$

where

$$
A=2 C_{c}\left(L_{1}+L_{2}+M\right) .
$$

The parasitic electrical coupling $C_{c}$ between two resonant circuits plays a critical role in (16) to determine the transmission zero frequencies. A larger value of $C_{c}$ pushes the two zero frequencies closer to the passband. However, the value of $C_{c}$ is difficult to change because of the predetermined coupling coefficient in (5) for a specified prototype filter response. In contrast, the transmission zero of $\omega_{Z 3}$ results from the series $L_{1}-C$ resonant branches in both feed structures and can be found as

$$
\omega_{Z 3}=\frac{1}{\sqrt{L_{1} C}} .
$$

Comparatively, adopting the antisymmetrical feed structure can provide two additional transmission zeros than the symmetrical construction to enhance the stopband rejection without sacrificing the passband response.

Planar transformers are commonly used in radio-frequency integrated circuits (RFICs) to play an important role for

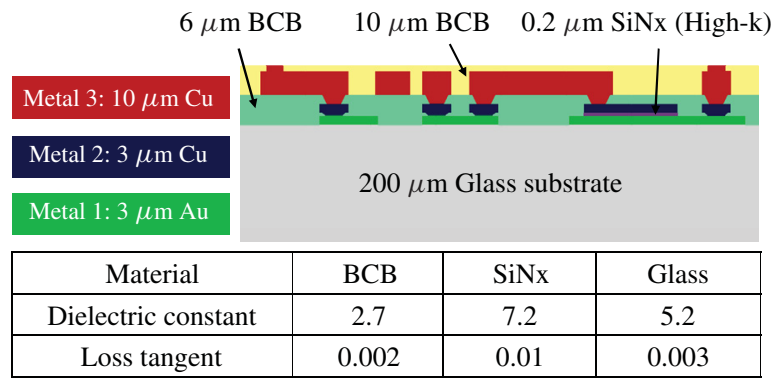

Fig. 4. Cross-sectional view and material parameters of the adopted IPD process on a glass substrate.

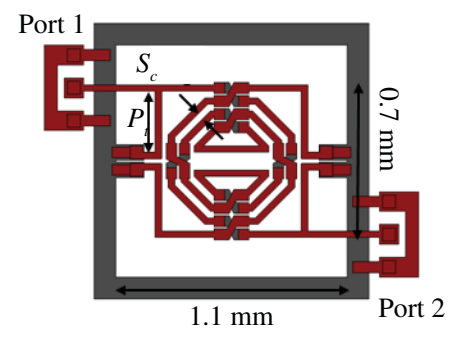

(a)

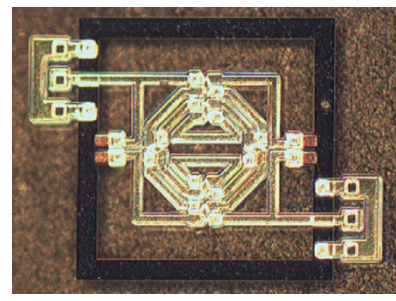

(b)
Fig. 5. Proposed transformer-coupled bandpass filter design using glass IPD technology. (a) Top-view layout. (b) Top-view photograph.

impedance transformation, balanced-to-unbalanced conversion, power combining, and coupling [18]-[20]. A recent study [21] developed an on-chip monolithic transformer configuration with a higher individual coil self-resonant frequency, greater area efficiency, and more fully symmetrical structure. This paper implements the proposed transformer-coupled bandpass filter using the transformer configuration in [21] based on integrated circuit technology. Fig. 4 illustrates the cross-sectional view and material parameters of the adopted IPD process on a glass substrate. The attractive features include the ability to produce high- $Q$ inductors and highdensity capacitors while using the metal 3 of $10-\mu \mathrm{m}$ copper, thin-film process, and high- $\varepsilon_{r}$ dielectrics. In addition, passive components using this technology can be easily integrated with 3-D chip-stacking applications.

A second-order 0.1-dB equal-ripple Chebyshev bandpass filter using the antisymmetrical feed structure is designed at the center frequency of $2.8 \mathrm{GHz}$ with a fractional bandwidth of $15 \%$, in addition, the $Q_{u}$ value of a coil-type resonator is 22. The prototype element values used are $g_{1}=0.843, g_{2}=$ 0.622 , and $g_{3}=1.355$. The coupling coefficient $k$ and the external quality factor $Q_{e}$ estimated by calculation are 0.21 and 5.6, respectively. The insertion loss at the center frequency that can be evaluated by (9) is $1.9 \mathrm{~dB}$. The metal width of the coil with respect to this design is $30 \mu \mathrm{m}$. Notably, an increase in metal width can enlarge the $Q_{u}$ value of the coil-type resonator. However, it is at the cost of increasing the IPD area.

Fig. 5(a) and (b) shows a top view of the layout and a photograph, respectively, of the actual device using glass IPD technology. Notably, according to the layout plot, a coil turn spacing of $S_{c}=30 \mu \mathrm{m}$ and a tapped feed position of $P_{t}=$ $280 \mu \mathrm{m}$ are used to correspond to the estimated $k$ and $Q_{e}$ values. For structural symmetry and flexible control of the $k$ 
TABLE I

Comparison of Measured Specifications and Sizes Between the Designed Glass IPd BandPass Filter and

SEVERAL RECENT WORKS IN THE LiteraturE

\begin{tabular}{|c|c|c|c|c|c|c|c|c|}
\hline \multirow{2}{*}{ Reference } & \multirow{2}{*}{ Technology } & \multirow{2}{*}{$\begin{array}{c}\text { Frequency } \\
\text { range, } \boldsymbol{f}_{\mathbf{0}} \mathrm{GHz}\end{array}$} & \multirow{2}{*}{ Dimension } & \multirow{2}{*}{ Insertion loss } & \multirow{2}{*}{ Return loss } & \multicolumn{3}{|c|}{ Stopband rejection } \\
\hline & & & & & & $<0.5 f_{0}$ & At $2 f_{0}$ & At $3 f_{0}$ \\
\hline This paper & Glass IPD & $2.7-2.9 \mathrm{GHz}$ & $1.1 \mathrm{~mm} \times 0.7 \mathrm{~mm}$ & $<2.2 \mathrm{~dB}$ & $>17 \mathrm{~dB}$ & $>33 \mathrm{~dB}$ & $36 \mathrm{~dB}$ & $46 \mathrm{~dB}$ \\
\hline \multirow{2}{*}[22]{} & \multirow{2}{*}{ Organic substrate } & \multirow{2}{*}{$f_{0}=2.5 \mathrm{GHz}$} & $3.7 \mathrm{~mm} \times 3.2 \mathrm{~mm}$ & $3.9 \mathrm{~dB}$ at $f_{0}$ & $8.1 \mathrm{~dB}$ at $f_{0}$ & N.A. & N.A. & N.A. \\
\hline & & & $1.5 \mathrm{~mm} \times 1.5 \mathrm{~mm}$ & $1.9 \mathrm{~dB}$ at $f_{0}$ & $14 \mathrm{~dB}$ at $f_{0}$ & N.A. & N.A. & N.A. \\
\hline [23] & Organic substrate & $2-3.2 \mathrm{GHz}$ & $4 \mathrm{~mm} \times 4.3 \mathrm{~mm}$ & $<1.7 \mathrm{~dB}$ & $>12 \mathrm{~dB}$ & $>14 \mathrm{~dB}$ & N.A. & N.A. \\
\hline [24] & Organic substrate & $2.4-2.5 \mathrm{GHz}$ & $2.2 \mathrm{~mm} \times 1.8 \mathrm{~mm}$ & $<1.7 \mathrm{~dB}$ & $>17 \mathrm{~dB}$ & $>21 \mathrm{~dB}$ & $23 \mathrm{~dB}$ & $37 \mathrm{~dB}$ \\
\hline$[25]$ & Glass IPD & $f_{0}=2.4 \mathrm{GHz}$ & N.A. & $<2.6 \mathrm{~dB}$ & $>9 \mathrm{~dB}$ & $>28 \mathrm{~dB}$ & $25 \mathrm{~dB}$ & N.A. \\
\hline \multirow{2}{*}{ [26] } & \multirow{2}{*}{ Silicon IPD } & \multirow{2}{*}{$f_{0}=2.45 \mathrm{GHz}$} & $1.7 \mathrm{~mm} \times 1.5 \mathrm{~mm}$ & $<1.7 \mathrm{~dB}$ & \multirow{2}{*}{ N.A. } & $>30 \mathrm{~dB}$ & $53 \mathrm{~dB}$ & $41 \mathrm{~dB}$ \\
\hline & & & $1.35 \mathrm{~mm} \times 1.1 \mathrm{~mm}$ & $<1.5 \mathrm{~dB}$ & & $>24 \mathrm{~dB}$ & $28 \mathrm{~dB}$ & $43 \mathrm{~dB}$ \\
\hline
\end{tabular}

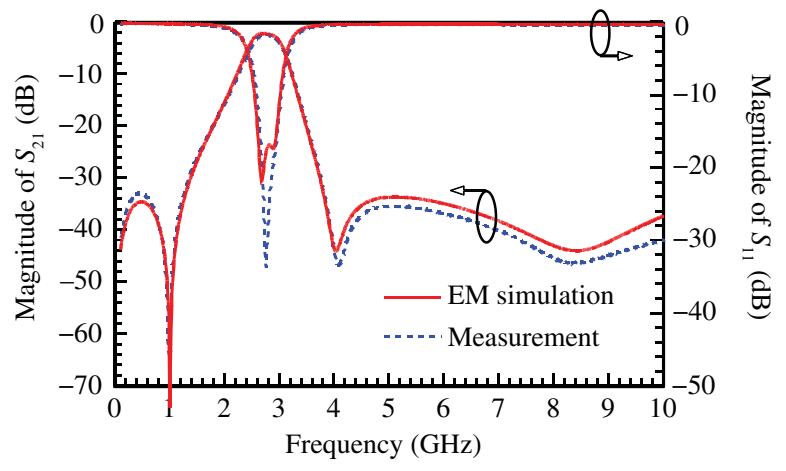

Fig. 6. Comparison of the magnitude of $S_{21}$ and $S_{11}$ between EM simulation and measurement for the designed transformer-coupled bandpass filter using glass IPD technology.

and $Q_{e}$ parameters, the transformer designed has an octagonal shape with the two parallel coils that are symmetrically interwound side by side on metal 3 . To serve as a common ground, a surrounding ground loop of square shape is also placed on metal 1 . The main effects of its proximity to the designed filter are to reduce the self-inductance of transformer, causing the center frequency to shift to a higher frequency and the bandwidth to become broader.

Measurements were carried out on an Agilent four-port vector network analyzer and a Cascade on-wafer probe station with ground-signal-ground (GSG) probes having a probe-tip pitch of $150 \mu \mathrm{m}$. The parasitic effects of GSG probing pads are included in the electromagnetic (EM) simulation by using Ansys-Ansoft HFSS. Fig. 6 compares the magnitudes of $S_{21}$ and $S_{11}$ between EM simulation and measurement for this second-order bandpass filter design, indicating a good agreement over a frequency range of up to $10 \mathrm{GHz}$. The insertion loss in the passband from 2.7 to $2.9 \mathrm{GHz}$ is approximately $2.2 \mathrm{~dB}$, which has a small deviation of 0.3 $\mathrm{dB}$ from the predicted value, in addition, the return loss in the passband exceeds $17 \mathrm{~dB}$. The tapped feed-lines shown in Fig. 5 result in a series resonant path starting from the tapped position to create a transmission zero at $4 \mathrm{GHz}$. The remaining two transmission-zero frequencies of 1 and 8.4 GHz come from the antisymmetrical feed structure, as mentioned earlier. The occupied area of the designed transformer-coupled bandpass filter, excluding the outer ground ring and test pads, is $1.1 \mathrm{~mm} \times 0.7 \mathrm{~mm}$.

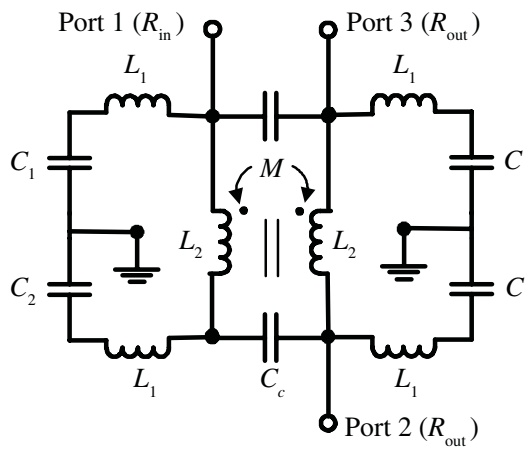

Fig. 7. Equivalent circuit of the transformer-coupled balun bandpass filter.

Table I compares the experimental results of the proposed bandpass filter design using glass IPD technology with those implemented on various kinds of package substrates or interposers in the recent literature [22]-[26]. The comparison reveals that our work has the advantages of a higher stopband rejection and a smaller component area.

\section{Creation And Matching of Balun BALANCED PORT}

The proposed transformer-coupled bandpass filter in Section II can easily integrate a balun function to convert an unbalanced signal into a balanced one without adjusting the filter size and shape, which is attributed to the following two reasons. First, the bandpass filter has a symmetric geometry.

Second, the bandpass filter uses magnetic coupling as the dominant coupling of the resonators. In practice, by simply combining the symmetric and antisymmetric feed structure in the output coil-type resonator as shown in Fig. 7, the feed-lines at ports 2 and 3 can then form a balanced port to effectively suppress the output common mode signals. However, this formation is accompanied by a disadvantage of changing the matching condition in the balanced port. According to literature, using the different coupling gaps between tapped line and input/output resonators [3] or controlling the direct current blocking capacitors [7] can resolve the mismatch problem.

Fig. 7 illustrates an equivalent circuit of the proposed transformer-coupled balun bandpass filter with the reference impedance of $R_{\text {in }}$ at port 1 and $R_{\text {out }}$ at ports 2 and 3 . The input 


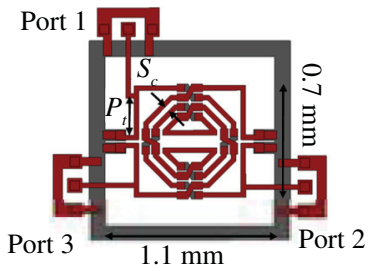

(a)

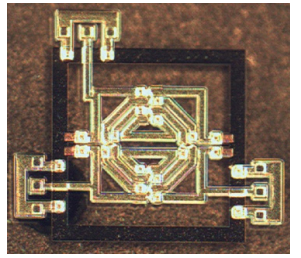

(b)
Fig. 8. Proposed balun bandpass filter design using glass IPD technology. (a) Top-view layout. (b) Top-view photograph.

and output coil-type resonators consist of a coil inductance $\left(L_{1}-L_{2}-L_{1}\right)$ with a different pair of capacitances of $C_{1}-C_{2}$ and $C-C$, respectively. This paper adopts two matching approaches for the proposed balun bandpass filter configuration as follows.

\section{A. Balanced Output Matching Approach}

An accurate tapped feed position determined by the required $Q_{e}$ based on the design parameters can achieve a desired matching in the passband. The input external quality factor $Q_{\text {e,in }}$ at port 1 in Fig. 7 appears to be identical to (6) under the condition of $C_{1}=C_{2}=C$. Furthermore, the output external quality factor $Q_{\mathrm{e} \text {,out }}$ at ports 2 and 3 can be found as

$$
Q_{\mathrm{e}, \text { out }}=R_{\mathrm{out}} \frac{\sqrt{2 C\left(2 L_{1}+L_{2}\right)^{3}}}{L_{2}^{2}}
$$

where $R_{\text {out }}$ denotes the reference impedance looking into the load termination at ports 2 and 3 in Fig. 7. Notably, $Q_{\mathrm{e}}$,in must equal $Q_{\mathrm{e} \text {,out }}$ for the bandpass filter design; this assumption implies that $R_{\text {out }}$ in (19) is twice the value of $R_{\text {in }}$ in (6). As mentioned earlier, $R_{\text {in }}$ equals $50 \Omega$ to match the source termination. However, $R_{\text {out }}$ is twice the value of $R_{\text {in }}$ as $100 \Omega$, resulting in a mismatch between the output ports (ports 2 and 3 ) and the load termination. A conventional approach reselects the output tapped feed position on the output coil resonator. However, such an approach is normally limited to the highdensity wiring transformer configuration because the tapped line can only connects to the peripheral portion of the coiltype resonator. In addition, while another matching approach adopts an impedance transformer between the output ports and the load termination, the transformer requires extra occupied area.

Fig. 8(a) and (b) shows the top-view layout and photograph, respectively, of such a transformer-coupled balun bandpass filter. Except for the additional port 3, the size and shape of the balun bandpass filter are the same as those of the previous bandpass filter shown in Fig. 5. Fig. 9 compares the magnitudes of $S_{D 1}$ and $S_{11}$ between EM simulation and measurement in a frequency range up to $10 \mathrm{GHz}$ for the implemented balun bandpass filter. The comparison shows good agreement. All ports are terminated by $50 \Omega$ in the simulation and measurement. The measured results indicate an insertion loss of less than $2.4 \mathrm{~dB}$ and a return loss of more than $14 \mathrm{~dB}$ in the passband. Notably, the aforementioned mismatch phenomenon in the output-balanced port only degrades the inband insertion and return losses slightly. Therefore, the in-band

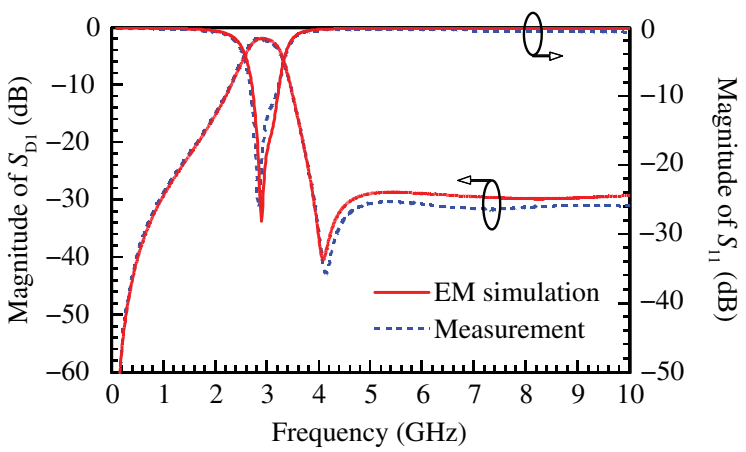

Fig. 9. Comparison of the magnitude of $S_{D 1}$ and $S_{11}$ between EM simulation and measurement for the designed balun bandpass filter using glass IPD technology.

performance is still quite similar to that of the bandpass-only filter design. Furthermore, the similarities also include a higher stopband transmission zero at $4 \mathrm{GHz}$. However, an obvious discrepancy is that the balun bandpass filter loses the stopband transmission zeros at the frequencies of 1 and $8.4 \mathrm{GHz}$.

The above similarities and dissimilarities can be explained by Fig. 7 whose $S$-parameters can be interpreted as a superposition result of Fig. 2(a) and (b). According to Fig. 3, the $S$-parameters corresponding to Fig. 2(a) and (b) have the same passband response and $4-\mathrm{GHz}$ transmission zero, but they differ in whether or not the existence of the 1 - and $8.4-\mathrm{GHz}$ transmission zeros. Such a difference eventually leads to the disappearance of the two transmission zeros at 1 and 8.4 GHz for the balun bandpass filter, as shown in Fig. 9. In mathematical point of view, the transmission zeros due to the output-balanced feed structure can be found by solving the following equation:

$Y_{\mathrm{D} 1}=\frac{1}{2}\left(Y_{21}-Y_{31}\right)=\frac{\left(Z_{1}+Z_{c}\right)\left[2 Z_{m} Z_{c}-\left(Z_{2}^{2}-Z_{m}^{2}\right)\right]}{2 Z_{c}\left[\left(Z_{1}+Z_{c}\right)\left(Z_{2}^{2}-Z_{m}^{2}\right)+Z_{1} Z_{2} Z_{c}\right]}$

where $Y_{D 1}$ stands for the differential to single-ended admittance parameter [27]-[29]. The solution for $Y_{D 1}=0$ has no real roots in the observed frequency range, verifying the disappearance of transmission zeros in the above inference.

\section{B. Single-Ended Input Matching Approach}

Reselecting the tapped feed position and the additionally required area of the impedance transformer poses a limitation to implementation. Conversely, providing the different values of capacitors $C_{1}$ and $C_{2}$ in the input coil-type resonator, as shown in Fig. 7 , can also lead to an impedance match between port 1 and source termination without implementation difficulties. In this manner, the $Q_{\text {e,out }}$ value and the tapped feed position in the output resonator are determined first for the required filter specification. Then, the input external quality $Q_{\text {e,in }}$ is set equal to $Q_{\text {e,out }}$, yielding the following equation:

$$
Q_{\mathrm{e}, \text { in }}=R_{\mathrm{in}} \frac{\sqrt{\left[\left(C_{1}+C_{2}\right)\left(2 L_{1}+L_{2}\right)\right]^{3} C_{1} C_{2}}}{\left[L_{2} C_{2}+L_{1}\left(C_{2}-C_{1}\right)\right]^{2}}=Q_{\mathrm{e}, \text { out }} .
$$




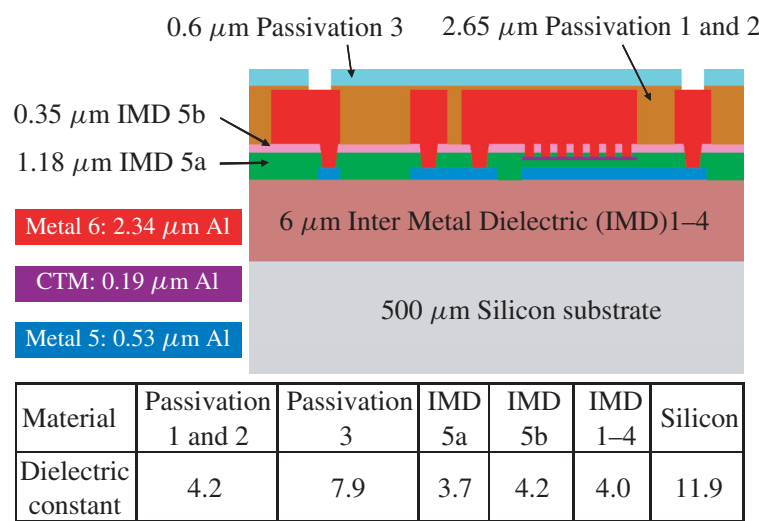

Fig. 10. Cross-sectional view and material parameters of the adopted $0.18 \mu \mathrm{m}$ CMOS process on a silicon substrate.

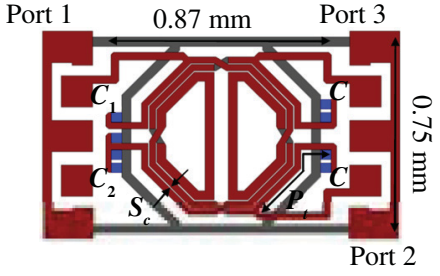

(a)

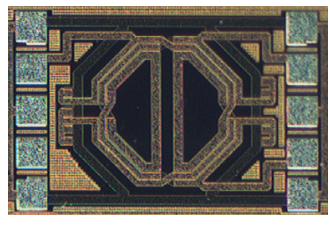

(b)
Fig. 11. Proposed balun bandpass filter design using $0.18 \mu \mathrm{m}$ CMOS technology. (a) Top-view layout. (b) Top-view photograph.

To ensure that the input and output resonators resonate at the same frequency, the capacitances have the following relation:

$$
\frac{1}{C_{1}}+\frac{1}{C_{2}}=\frac{2}{C}
$$

Satisfying the matching condition requires the appropriate $C_{1}$ and $C_{2}$ to meet (21) and (22) simultaneously.

Next, the proposed single-ended input matching approach is implemented on a balun bandpass filter for the $3.1-4.85 \mathrm{GHz}$ direct-sequence ultrawideband (DS-UWB) using $0.18 \mu \mathrm{m}$ CMOS process with a substrate cross-sectional view in Fig. 10. The resonator used comprises a coil inductor on the metal 6 and two metal-insulator-metal capacitors using the capacitor top metal (CTM) and metal 5 layers. In this design, a secondorder Chebyshev bandpass filter with $0.5 \mathrm{~dB}$ ripple level is designed with a fractional bandwidth of $40 \%$. The prototype element values used are $g_{1}=1.403, g_{2}=0.707$, and $g_{3}=$ 1.984. The estimated coupling coefficient and external quality factor are $k=0.4$ and $Q_{e}=3.5$, respectively. The unloaded quality factor $Q_{u}$ of the coil-type resonator is 7.6 and results in a calculated insertion loss of $3 \mathrm{~dB}$ by using (9).

Fig. 11(a) and (b) displays the top-view layout and photograph, respectively, of the designed CMOS balun bandpass filter. The required coil turn spacing $S_{c}$ for a $40 \%$ bandwidth is $4 \mu \mathrm{m}$, and the tapped feed position $P_{t}$ at both the input and the output resonators is $420 \mu \mathrm{m}$. In addition, the series capacitors at both ends present the values of $C_{1}=0.8 \mathrm{pF}, C_{2}=2.4 \mathrm{pF}$, and $C=1.2 \mathrm{pF}$ for the single-ended input matching approach to satisfy (21) and (22). The EM simulation results correlate well with the measurement results, as shown in Fig. 12.

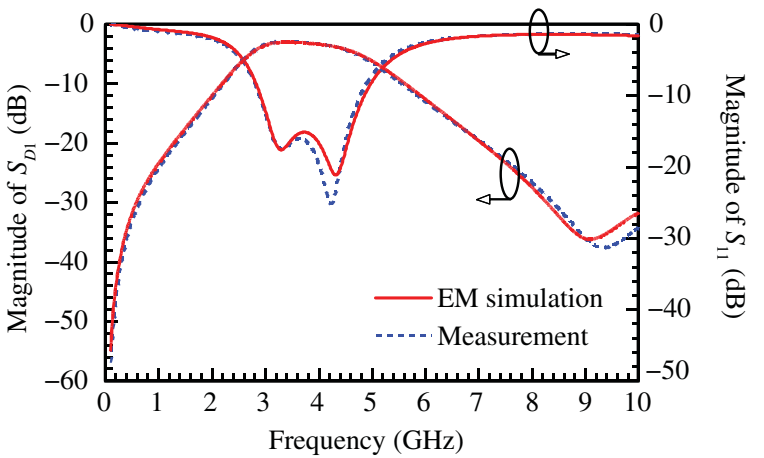

Fig. 12. Comparison of the magnitude of SD1 and S11 between EM simulation and measurement for the designed balun bandpass filter using $0.18 \mu \mathrm{m}$ CMOS technology.

In the passband, the measured insertion loss ranges from 2.8 to $5.5 \mathrm{~dB}$, while the return loss is better than $18 \mathrm{~dB}$. The transmission zero at $9.3 \mathrm{GHz}$ comes from the series resonant path starting at the tapped position. The occupied area of the designed CMOS transformer-coupled balun bandpass filter, excluding the outer ground ring and test pads, is $0.8 \mathrm{~mm} \times 0.7 \mathrm{~mm}$.

\section{BANDWIDTH-DEPENDENT CMRR}

The CMRR is conventionally adopted to evaluate the balance performance of a balun [30]. It is defined as the ratio between the differential-mode and common-mode transmission-coefficient magnitude. At the center frequency as expressed in (1), CMRR can be derived as (23) for the proposed equivalent circuit in Fig. 7

$$
\begin{aligned}
& C M R R\left(\omega_{0}\right) \\
& \approx\left|\frac{2 M C_{2}\left(\omega_{0} C-j G_{\text {out }}\right)}{\omega_{0}^{3} M^{2} C_{2} C_{c} C+j 2 G_{\text {out }} L_{2} C_{c}\left(\omega_{0}^{2} L_{1} C-1\right)\left(\frac{C_{2}}{C}-1\right)}\right|
\end{aligned}
$$

where $G_{\text {out }}=1 / R_{\text {out }}$. Notably, the single-ended input matching approach requires the different values of $C_{2}$ and $C$ and results in degradation in CMRR. For a specific requirement of equality between $C_{2}$ and $C$ as the previous glass IPD balun bandpass filter design in Fig. 8, CMRR can then be obtained in terms of the magnetic and electrical coupling coefficients as

$$
\begin{aligned}
C M R R\left(\omega_{0}\right) & =\left|\frac{2}{\omega_{0}^{2} M C_{c}} \cdot \frac{\left(\omega_{0} C-j G_{\mathrm{out}}\right)}{\omega_{0} C}\right| \\
& =\left|\frac{1}{k_{\mathrm{M}} k_{\mathrm{E}}} \cdot \frac{\left(\omega_{0} C-j G_{\mathrm{out}}\right)}{2 \omega_{0} C}\right| .
\end{aligned}
$$

Fig. 13 compares the CMRR simulation results of the balun bandpass filter by varying either the electrical coupling coefficient $k_{E}$ or the magnetic coupling coefficient $k_{M}$. Fig. 13(a) shows the CMRR results with three different $k_{E}$ values of $0.01,0.02$, and 0.04 and the same $k_{M}$ value of 0.15 . It can be seen from Fig. 13(a) that the peak CMRR increases with decreasing $k_{E}$. In a similar fashion, Fig. 13(b) demonstrates the CMRR results with three different $k_{M}$ values of $0.15,0.30$, and 0.60 and the same $k_{E}$ value of 0.15 . One can observe from 


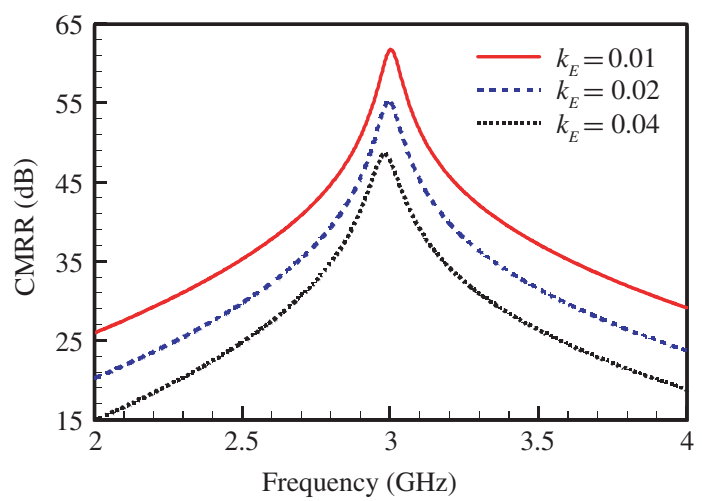

(a)

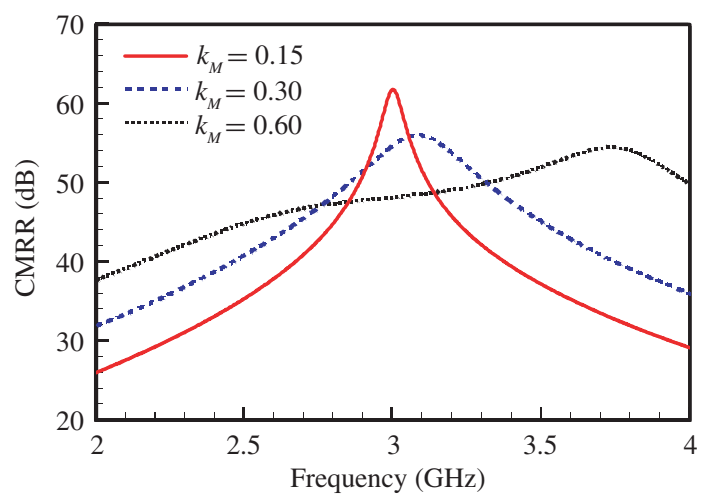

(b)

Fig. 13. Comparison of the CMRR for the balun bandpass filter design with different electrical and magnetic coupling coefficients. (a) CMRR with varying $k_{E}$. (b) CMRR with varying $k_{M}$.

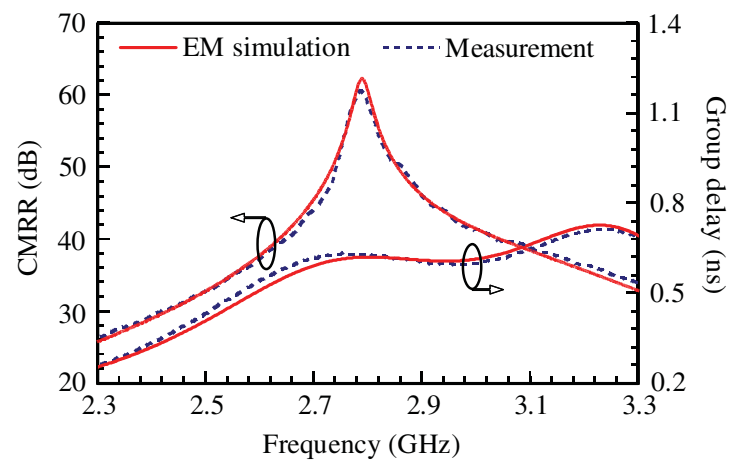

Fig. 14. Comparison of the CMRR and group delay between EM simulation and measurement for the designed balun bandpass filter using glass IPD technology.

Fig. 13(b) that the peak CMRR moves to a higher frequency as $k_{M}$ increases. Therefore, the optimal CMRR within a narrow frequency band of interest can be achieved by selecting an appropriate value of $k_{M}$ and an as small as possible value of $k_{E}$.

Since the mutual inductance $M$ dominates the coupling for the high-density wiring transformer-coupled configuration, the coupling coefficient in (7) can be approximated as

$$
k=\frac{\Delta}{\sqrt{g_{1} g_{2}}} \approx k_{\mathrm{M}}
$$

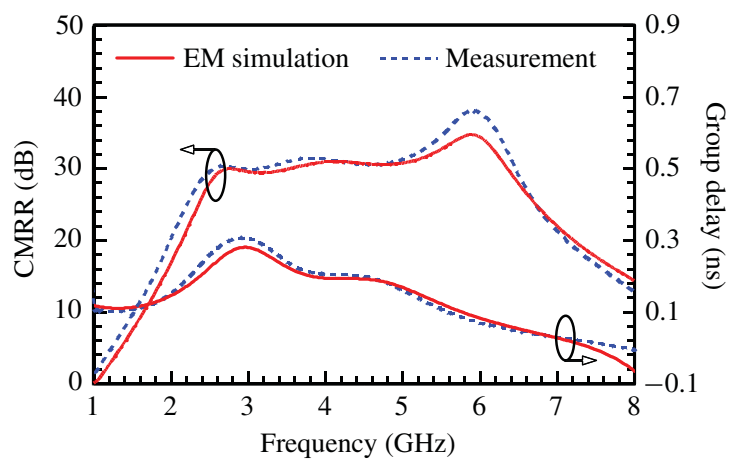

Fig. 15. Comparison of the CMRR and group delay between EM simulation and measurement for the designed balun bandpass filter using $0.18 \mu \mathrm{m}$ CMOS technology.

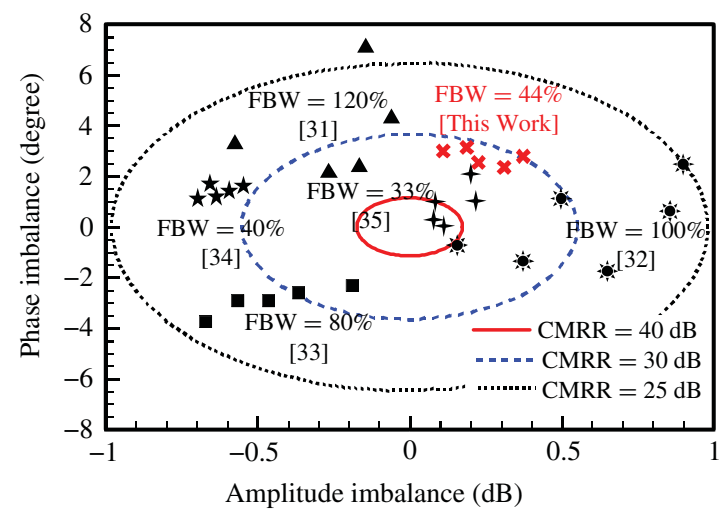

Fig. 16. Comparison of the measured amplitude and phase imbalances between the proposed CMOS balun bandpass filter design and the available balun-only integrated circuit designs.

Incorporating (25) into (24) and further rearranging leads to

$$
\operatorname{CMRR}\left(\omega_{0}\right) \approx\left|\frac{\sqrt{g_{1} g_{2}}}{\Delta \cdot k_{E}} \cdot \frac{\left(\omega_{0} C-j G_{\mathrm{out}}\right)}{2 \omega_{0} C}\right|
$$

where $\Delta$ denotes the fractional bandwidth of the balun bandpass filter. Equation (26) can provide an efficient means of optimizing the CMRR by designing the operation bandwidth $\Delta$ precisely and minimizing the electrical coupling coefficient $k_{E}$ between the interwound coil-type resonators during filter construction.

As a result, Fig. 14 compares the CMRR and group delay between EM simulation and measurement in the passband and its vicinity for the designed balun bandpass filter on the glass IPD substrate with a top-view layout and photograph shown in Fig. 8(a) and (b), respectively. The comparison reveals satisfactory agreement. The measured CMRR for this design exceeds $44 \mathrm{~dB}$ in the passband from 2.7 to $2.9 \mathrm{GHz}$, which represents an outstanding balance performance, the measured value of group delay is around $0.62 \mathrm{~ns}$.

Fig. 15 compares the CMRR and group delay between EM simulation and measurement for the designed CMOS balun bandpass filter with a top-view layout and photograph shown in Fig. 11(a) and (b), respectively. According to Fig. 15, the measured CMRR for this design is better than $30 \mathrm{~dB}$ and the group delay ranges from 0.18 to $0.28 \mathrm{~ns}$ over the passband from 3.1 to $4.85 \mathrm{GHz}$. 
TABLE II

Comparison of Measured Specifications and Sizes Between the Designed Glass IPD Balun Bandpass Filter and SEVERAL RECENT WORKS IN THE Literature

\begin{tabular}{|c|c|c|c|c|c|c|c|}
\hline Reference & Technology & Frequency range $(\mathrm{GHz})$ & Dimension & $\begin{array}{c}\text { Insertion } \\
\text { loss }\end{array}$ & $\begin{array}{c}\text { Amplitude } \\
\text { imbalance }\end{array}$ & $\begin{array}{c}\text { Phase } \\
\text { imbalance }\end{array}$ & CMRR \\
\hline IPD Balun BPF & Glass IPD & $2.7-2.9$ & $1.1 \mathrm{~mm} \times 0.7 \mathrm{~mm}$ & $<2.2 \mathrm{~dB}$ & $<0.08 \mathrm{~dB}$ & $<0.5^{\circ}$ & $>44 \mathrm{~dB}$ \\
\hline$[2]$ & LTCC & $3.3-3.9$ & $3 \mathrm{~mm} \times 1.6 \mathrm{~mm}$ & $<1.8 \mathrm{~dB}$ & $<0.7 \mathrm{~dB}$ & $<5^{\circ}$ & NA \\
\hline$[3]$ & Silicon IPD & $2.4-2.5$ & $1.5 \mathrm{~mm} \times 1.5 \mathrm{~mm}$ & $<2.2 \mathrm{~dB}$ & $<0.5 \mathrm{~dB}$ & $<3^{\circ}$ & $>30 \mathrm{~dB}$ \\
\hline$[4]$ & $\begin{array}{c}\text { PCB } \\
\text { Taconic } \\
\text { RF-60A) }\end{array}$ & $2.43-2.47 \mathrm{GHz}$ & $16 \mathrm{~mm} \times 18 \mathrm{~mm}$ & $<2.5 \mathrm{~dB}$ & $<0.5 \mathrm{~dB}$ & $<5^{\circ}$ & N.A. \\
\hline$[5]$ & LTCC & $2.3-2.6 \mathrm{GHz}$ & $4.2 \mathrm{~mm} \times 4.8 \mathrm{~mm}$ & N.A. & $<0.3 \mathrm{~dB}$ & $<1^{\circ}$ & N.A. \\
\hline$[6]$ & LTCC & $2.4-2.5 \mathrm{GHz}$ & $2.5 \mathrm{~mm} \times 2 \mathrm{~mm}$ & $<2 \mathrm{~dB}$ & N.A. & N.A. & $>30 \mathrm{~dB}$ \\
\hline$[7]$ & LTCC & $2.4-2.5 \mathrm{GHz}$ & $2 \mathrm{~mm} \times 1.2 \mathrm{~mm}$ & $<1.7 \mathrm{~dB}$ & $<0.3 \mathrm{~dB}$ & $<3^{\circ}$ & N.A. \\
\hline$[8]$ & LTCC & $1.45-1.75 \mathrm{GHz}$ & $2 \mathrm{~mm} \times 1.25 \mathrm{~mm}$ & $<1.1 \mathrm{~dB}$ & $<0.6 \mathrm{~dB}$ & $<5^{\circ}$ & N.A. \\
\hline$[9]$ & LTCC & $2.3-2.6 \mathrm{GHz}$ & $2 \mathrm{~mm} \times 1.2 \mathrm{~mm}$ & $<3.5 \mathrm{~dB}$ & $<0.6 \mathrm{~dB}$ & $<12.5^{\circ}$ & N.A. \\
\hline$[10]$ & $\begin{array}{c}\text { PCB } \\
\text { (BSrTiO3) }\end{array}$ & $2.4-2.5 \mathrm{GHz}$ & $2 \mathrm{~mm} \times 1.7 \mathrm{~mm}$ & $<2.2 \mathrm{~dB}$ & $<0.9 \mathrm{~dB}$ & $<1.5^{\circ}$ & $>26 \mathrm{~dB}$ \\
\hline
\end{tabular}

TABLE III

Comparison of Measured Specifications and Sizes Between the Designed Glass ipd Balun Bandpass Filter and SEVERAL RECENT WORKS IN THE LITERATURE

\begin{tabular}{|c|c|c|c|c|c|c|}
\hline Reference & Technology & Frequency range $(\mathrm{GHz})$ & Dimension & Insertion loss & $\begin{array}{l}\text { Amplitude } \\
\text { imbalance }\end{array}$ & Phase imbalance \\
\hline CMOS Balun BPF & CMOS & $3.1-4.85(44 \%)$ & $0.8 \mathrm{~mm} \times 0.7 \mathrm{~mm}$ & $2.8-5.5 \mathrm{~dB}$ & $<0.5 \mathrm{~dB}$ & $<3^{\circ}$ \\
\hline$[31]$ & Silicon IPD & $1.4-5.6(120 \%)$ & $1.1 \mathrm{~mm} \times 2.1 \mathrm{~mm}$ & $2-5 \mathrm{~dB}$ & $\begin{array}{c}<0.6 \mathrm{~dB} \text { at } \\
(1.4-3.5 \mathrm{GHz})\end{array}$ & $\begin{array}{c}<7.8^{\circ} \text { at } \\
(1.4-3.5 \mathrm{GHz})\end{array}$ \\
\hline$[32]$ & Silicon above-IC & $5-15(100 \%)$ & $1.7 \mathrm{~mm} \times 1.05 \mathrm{~mm}$ & $1-5 \mathrm{~dB}$ & $\begin{array}{c}<1 \mathrm{~dB} \text { at } \\
(5-12 \mathrm{GHz})\end{array}$ & $\begin{array}{c}<2.6^{\circ} \text { at } \\
(5-12 \mathrm{GHz})\end{array}$ \\
\hline [33] & MCM-D alumina & $3.6-8.4(80 \%)$ & $11 \mathrm{~mm} \times 1.76 \mathrm{~mm}$ & $0.5-1.1 \mathrm{~dB}$ & $<0.9 \mathrm{~dB}$ & $<3.3^{\circ}$ \\
\hline$[34]$ & MEMS & $4.3-6.5(40 \%)$ & $0.4 \mathrm{~mm} \times 0.5 \mathrm{~mm}$ & $1.1-3 \mathrm{~dB}$ & $<0.7 \mathrm{~dB}$ & $<1.2^{\circ}$ \\
\hline [35] & Silicon IPD & $1.5-2.1(33 \%)$ & $1.65 \mathrm{~mm} \times 1.5 \mathrm{~mm}$ & $0.8 \mathrm{~dB}$ & $<0.2 \mathrm{~dB}$ & $<2^{\circ}$ \\
\hline [36] & $0.35 \mu \mathrm{m} \mathrm{SiGe}$ & $6-10 \mathrm{GHz}(50 \%)$ & $0.25 \mathrm{~mm} \times 0.5 \mathrm{~mm}$ & $3.9-5 \mathrm{~dB}$ & $<1 \mathrm{~dB}$ & $<4^{\circ}$ \\
\hline
\end{tabular}

Table II compares the experimental results of the proposed balun bandpass filter design using glass IPD technology with those of recent literature in terms of integrating a bandpass filter and a balun into one component [2]-[10]. According to Table II, our work achieves a similar insertion loss but a superior CMRR level in the passband when compared to the other works. This significant CMRR performance is resulted from the precisely controlled bandwidth and the adoption of glass IPD technology that has a smaller electrical coupling coefficient than the use of LTCC and PCB technologies. In addition, the occupied area is the smallest one.

Table III compares the proposed CMOS balun design with a bandwidth controlled by the integrated bandpass filter to the available balun-only designs using CMOS, silicon IPD, microelectromechanical systems (MEMS) and multi-chip moduledeposited (MCM-D) technologies [31]-[36]. Notably, in Table III, the fractional bandwidths are defined for return loss more than $10 \mathrm{~dB}$. Due to the high conductor loss and the low silicon resistivity in standard CMOS process, our work results in a slightly larger insertion loss. However, it achieves an optimal CMRR level over the required fractional bandwidth. Fig. 16 illustrates the relation between the CMRR and the fractional bandwidth. The symbols present the frequency dependence of CMRR for individual cases in Table III. This figure generally reveals that a more wideband balun design is more likely to degrade the CMRR level and its variation in frequency within the operating bandwidth. The proposed bandwidth-controlled method can optimize the CMRR level in a specified fractional bandwidth with a smaller variation than the other ones in [31]-[35]. The resultant CMRR level is more than $30 \mathrm{~dB}$ in the 3.1-4.85 GHz DS-UWB band with less than $2 \mathrm{~dB}$ variation.

\section{CONClusion}

This paper has developed a transformer-coupled bandpass filter design based on integrated circuit technology. Advantages of the proposed filter design include an extremely small size and ease of integrating a balun without adjusting the size and shape in configuration and degrading the passpand loss and bandwidth in performance of the primary bandpass filter design. Consequently, the proposed balun bandpass filter achieves an overall lower insertion loss than that of a circuit structure with separate bandpass and balun components. In addition, the CMRR has a relevant connection with the fractional bandwidth of the primary bandpass filter design. Moreover, the narrow-band design using glass IPD technology provides an excellent balance performance with a significantly higher CMRR level than that of previous works. Furthermore, the wideband design using 
$0.18 \mu \mathrm{m}$ CMOS technology can obtain an optimal CMRR level by precisely controlling the filter bandwidth to match the operating frequency range of the balun.

\section{ACKNOWLEDGMENT}

The authors would like to thank National Chip Implementation Center (CIC), Taipei, Taiwan, for chip fabrication, for providing the glass integrated passive device, and for complementary metal-oxide-semiconductor foundry service.

\section{REFERENCES}

[1] M. Rotaru, Y. Y. Lim, H. Kuruveettil, R. Yang, A. P. Popov, and C.-P. Chua, "Implementation of packaged integrated antenna with embedded front end for bluetooth applications," IEEE Trans. Adv. Packag., vol. 31, no. 3, pp. 558-567, Aug. 2008 .

[2] S. Sakhnenko, D. Orlenko, K. Markov, and A. Yatsenko, "Low profile LTCC balanced filter based on a lumped elements balun for WiMAX applications," in Proc. IEEE MTT-S Int. Microw. Symp. Dig., Atlanta, GA, Jun. 2008, pp. 1111-1114.

[3] K. Liu, R. Frye, and R. Emigh, "Band-pass-filter with balun function from IPD technology," in Proc. 58th Electron. Comp. Technol. Conf., Lake Buena Vista, FL, 2008, pp. 718-723.

[4] E.-Y. Jung and H.-Y. Hwang, "A balun-BPF using a dual mode ring resonator," IEEE Microw. Wireless Compon. Lett., vol. 17, no. 9, pp. 652-654, Sep. 2007.

[5] L. K. Yeung and K.-L. Wu, "An LTCC balanced-to-unbalanced extracted-pole bandpass filter with complex load," IEEE Trans. Microw. Theory Tech., vol. 54, no. 4, pp. 1512-1518, Jun. 2006

[6] C.-C. Chuang and C.-L. Wang, "Design of three-pole single-to-balanced bandpass filters," in Proc. Eur. Microw. Conf., Manchester, U.K., 2006, pp. 1193-1196.

[7] K.-T. Chen and S.-J. Chung, "A novel compact balanced-to-unbalanced low-temperature co-fired ceramic bandpass filter with three coupled lines configuration," IEEE Trans. Microw. Theory Tech., vol. 56, no. 7, pp. 1714-1720, Jul. 2008.

[8] C.-L. Tsai and Y.-S. Lin, "Analysis and design of new single-tobalanced multicoupled line bandpass filters using low-temperature cofired ceramic technology," IEEE Trans. Microw. Theory Tech., vol. 56, no. 12, pp. 2902-2912, Dec. 2008.

[9] M. Tamura, T. Ishizaki, and M. Hoft, "Design and analysis of vertical split ring resonator and its application to unbalanced-balanced filter," IEEE Trans. Microw. Theory Tech., vol. 58, no. 1, pp. 157-164, Jan. 2010.

[10] J. C. Park, J. Y. Park, and S. G. Yoon, "PCB embedded compact balanced filter with coupled LC resonators," in Proc. 59th Electron. Comp. Technol. Conf., 2009, pp. 1977-1982.

[11] C.-H. Wu, C.-H. Wang, S.-Y. Chen, and C.-H. Chen, "Balanced-tounbalanced bandpass filters and the antenna application," IEEE Trans. Microw. Theory Tech., vol. 56, no. 11, pp. 2474-2482, Nov. 2008.

[12] C.-H. Chen, C.-H. Huang, T.-S. Horng, S.-M. Wu, C.-T. Chiu, C.-P. Hung, J.-Y. Li, and C.-C. Chen, "Very compact transformer-coupled balun-integrated bandpass filter using integrated passive device technology on glass substrate," in Proc. IEEE MTT-S Int. Microw. Symp. Dig., Anaheim, CA, May 2010, pp. 1372-1375.

[13] J.-S. Hong and M. J. Lancaster, Microstrip Filters for RF/Microwave Applications. New York: Wiley, 2001, pp. 109-272.

[14] G. L. Matthaei, L. Young, and E. M. T. Jones, Microwave Filters, Impedance Matching Networks, and Coupling Structures. New York: McGraw-Hill, 1964, ch. 8.

[15] R. W. Rhea, HF Filter Design and Computer Simulation. Atlanta, GA: Noble Publishing, 1994, ch. 5.

[16] C.-M. Tsai, S.-Y. Lee, and C.-C. Tsai, "Performance of a planar filter using a $0^{\circ}$ feed structure," IEEE Trans. Microw. Theory Tech., vol. 50, no. 10 , pp. 2362-2367, Oct. 2002.

[17] C.-F. Chang and S.-J. Chung, "Bandpass filter of serial configuration with two finite transmission zeros using LTCC technology," IEEE Trans. Microw. Theory Tech., vol. 53, no. 7, pp. 2383-2388, Jul. 2005.

[18] J. R. Long, "Monolithic transformers for silicon RF IC design," IEEE J. Solid State Circuits, vol. 35, no. 9, pp. 1368-1382, Sep. 2000.

[19] K. H. An, O. Lee, H. Kim, D. H. Lee, J. Han, K. S. Yang, Y. Kim, J. J. Chang, W. Woo, C.-H. Lee, H. Kim, and J. Laskar, "Power-combining transformer techniques for fully-integrated CMOS power amplifier," IEEE J. Solid State Circuits, vol. 43, no. 5, pp. 1064-1075, May 2008.
[20] C.-H. Huang, T.-C. Wei, T.-S. Horng, J.-Y. Li, C.-C. Chen, C.-C. Wang, C.-T. Chiu, and C.-P. Hung, "Design and modeling of planar transformerbased integrated passive devices for wireless applications," in Proc. 59th Electron. Comp. Technol. Conf., San Diego, CA, 2009, pp. 516-521.

[21] C. C. Lim, K. S. Yeo, K. W. Chew, A. Cabuk, J.-M. Gu, S. F. Lim, C. C. Boon, and M. A. Do, "Fully symmetrical monolithic transformer (true 1:1) for silicon RFIC," IEEE Trans. Microw. Theory Tech., vol. 56, no. 10 , pp. 2301-2311, Oct. 2008.

[22] G.-A. Lee, M. A. Megahed, and F. De Flaviis, "Low-cost compact spiral inductor resonator filters for system-in-a-package," IEEE Trans. Adv. Packag., vol. 28, no. 4, pp. 761-771, Nov. 2005.

[23] U.-M. Jow, Y.-J. Lai, C.-L. Weng, C.-S. Chen, and C.-S. Shyu, "Functional embedded RF circuits on multilayer printed wiring board (PWB) process," in Proc. 55th Electron. Comp. Technol. Conf., 2005, pp. 16341641.

[24] S. J. Lee and J. Y. Park, "PCB embedded $2.4 \mathrm{GHz}$ compact bandpass filter with two finite transmission zeros," in Proc. Asia Pacific Microw. Conf., Bangkok, Thailand, 2007, pp. 1-4.

[25] V. Sridharan, S. Min, V. Sundaram, V. Sukumaran, S. Hwang, H. Chan, F. Liu, C. Nopper, and R. Tummala, "Design and fabrication of bandpass filters in glass interposer with through-package-vias (TPV)," in Proc. 60th Electron. Comp. Technol. Conf., Las Vegas, NV, 2010, pp. 530535.

[26] R. C. Frye, K. Liu, G. Badakere, and L. Yaojian, "A hybrid coupledresonator bandpass filter topology implemented on lossy semiconductor substrates," in Proc. IEEE MTT-S Int. Microw. Symp. Dig., Honolulu, HI, Jun. 2007, pp. 1757-1760.

[27] Touchstone File Format Specification, Agilent Technologies, Santa Clara, CA, 2009.

[28] D. E. Bockelman and W. R. Eisenstadt, "Pure-mode network analyzer for on-wafer measurements of mixed-mode S-parameters of differential circuits," IEEE Trans. Microw. Theory Tech., vol. 45, no. 7, pp. 10711077, Jul. 1997.

[29] A. Ferrero and M. Pirola, "Generalized mixed-mode S-parameters," IEEE Trans. Microw. Theory Tech., vol. 54, no. 1, pp. 458-463, Jan. 2006.

[30] W. R. Eisenstadt, B. Stengel, and B. M. Thompson, Microwave Differential Circuit Design Using Mixed-Mode S-Parameters. Boston, MA: Artech House, 2006, ch. 5.

[31] Y. J. Yoon, Y. Lu, R. C. Frye, and P. R. Smith, "Spiral transmission-line baluns for RF multichip module packages," IEEE Trans. Adv. Packag., vol. 22, no. 3, pp. 332-336, Aug. 1999.

[32] S. Xiao, O. Dupuis, D. Linten, G. Carchon, P. Soussan, S. Decoutere, D. R. Walter, and E. Beyne, "High- $Q$ above-IC inductors using thin-film wafer-level packaging technology demonstrated on 90-nm RF-CMOS 5-GHz VCO and 24-GHz LNA," IEEE Trans. Adv. Packag., vol. 29 , no. 4, pp. 810-817, Nov. 2006.

[33] M. Chongcheawchamnan, C. Y. Ng, M. S. Aftanasar, I. D. Robertson, and J. S. Minalgene, "A broadband CPW Marchand balun using a photoimagable multilayer thick-film process," Electron. Lett., vol. 37, no. 20, pp. 1228-1229, Sep. 2001.

[34] Z. Wu and X. Li, "On-chip micromachined solenoid balun for RF-SoC applications," Electron. Lett., vol. 45, no. 8, pp. 409-411, Apr. 2009.

[35] K.-Y. Chen, B.-X. Fang, and H.-H. Yeh, "IPD broadband balun design for GSM applications," in Proc. IEEE Electr. Des. Adv. Packag. Syst. Symp., Singapore, Dec. 2010, pp. 1-4.

[36] S.-C. Tseng, C. Meng, C.-H. Chang, and G.-W. Huang, "SiGe HBT Gilbert downconverter with an integrated miniaturized Marchand balun for UWB applications," in Proc. IEEE MTT-S Int. Microw. Symp. Dig., Honolulu, HI, Jun. 2007, pp. 2141-2144.

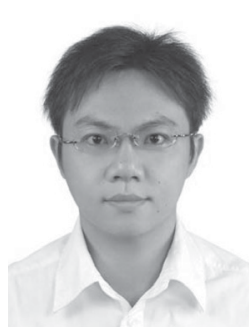

Chien-Hsun Chen (S'11) was born in Kaohsiung, Taiwan, on January 30, 1981. He received the B.S.E.E degree from Yuan-Ze University, Taoyuan, Taiwan, and the M.S.E.E. degree from I-Shou University, Kaohsiung, in 2003 and 2005, respectively. $\mathrm{He}$ is currently pursuing the Ph.D. degree with the Electrical Engineering Department, National Sun Yat-Sen University, Kaohsiung.

His current research interests include the design and analysis of embedded and integrated passive components for wireless communication. 


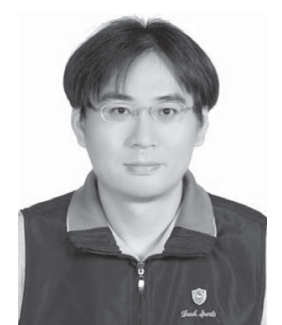

Chien-Hsiang Huang (S'09) was born in Kaohsiung, Taiwan, on August 24, 1978. He received the B.S.E.E. and M.S.E.E. degrees from National Sun Yat-Sen University, Kaohsiung, in 2000 and 2002, respectively. He is currently pursuing the Ph.D. degree in electrical engineering with National Sun Yat-Sen University.

His current research interests include design and modeling of radio frequency/microwave integrated passive devices.

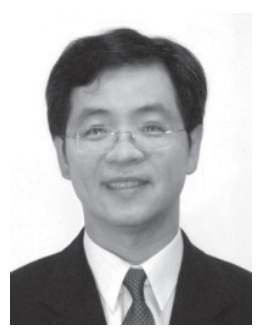

Tzyy-Sheng Horng (S'88-M'92-SM'05) was born in Taichung, Taiwan, on December 7, 1963. He received the B.S.E.E. degree from National Taiwan University, Taipei, Taiwan, in 1985, and the M.S.E.E. and Ph.D. degrees from the University of California, Los Angeles, in 1990 and 1992, respectively.

He has been with the Department of Electrical Engineering, National Sun Yat-Sen University, Kaohsiung, Taiwan, since August 1992, where he was the Director of the Telecommunication Research and Development Center from 2003 to 2008 and the Director of the Institute of Communications Engineering from 2004 to 2007, where he is currently a Professor. He has authored or co-authored over 100 technical publications published in IEEE journals and conference proceedings. He holds over ten patents. His current research interests include radio frequency (RF) and microwave integrated circuits and components, RF signal integrity for wireless systems-in-package, and digitally assisted RF technologies.

Dr. Horng has served on several technical program committees of international conferences including the International Association of Science and Technology for Development International Conference on Wireless and Optical Communications, the IEEE Region 10 International Technical Conference, the IEEE International Workshop on Electrical Design of Advanced Packaging and Systems, the Asia-Pacific Microwave Conference, the IEEE Radio and Wireless Symposium, and the Electronic Components and Technology Conference. He was the recipient of the Young Scientist Award presented by the International Union of Radio Science in 1996, the Industry-Education Cooperation Award presented by the Ministry of Education, Taiwan, in 1998, and the Distinguished Electrical Engineer Award presented by the Chinese Institute of Electrical Engineering, Taiwan, in 2010. 